Pacific Journal of Mathematics

ON THE PROJECTIVE NORMALITY OF SOME VARIETIES OF 


\title{
ON THE PROJECTIVE NORMALITY OF SOME VARIETIES OF DEGREE 5
}

\author{
AKIRA OHBUCHI
}

\begin{abstract}
We give some sufficient conditions for projective normality of complete non-singular varieties of degree five. And we prove that every complete non-singular surfaces of degree five embedded by a complete linear system is projectively normal.
\end{abstract}

Introduction. Let $X$ be a complete non-singular variety over an algebraically closed field, and let $L$ be an ample line bundle on $X$. The classification of some $(X, L)$ is found in Fugita's papers (Fujita [1], [2], [3], [4]). In this paper, we consider the projective normality of $(X, L)$ and the defining equations. This problem is trivial in the case of $\left(D^{n}\right)=1,2$ where $n=\operatorname{dim} X$ and $\mathscr{L}=\mathscr{O}(D)$. If $\left(D^{n}\right)=3$, then $(X, L)$ is projectively normal and the ideal is generated by degree 2 and 3 (X.X.X. [11]). If $\left(D^{n}\right)=4$, then $(X, L)$ is projectively normal and the ideal is generated by degree 2 and 3 (Swinnerton-Dyer [10]). So we consider the case of $\left(D^{n}\right)=5$. In this paper we give some sufficient conditions for projective normality of varieties of degree 5 and give the generator of the defining ideal. The main part of this paper is the case of $\left(D^{n}\right)=5$ and $\Delta(X, L)=2$ (other cases are clearly obtained by Fujita's theory). This is a non-degenerate and non-singular variety of codimension 2 in some projective space $\mathbb{P}^{N}$. On the other hand, the following conjecture is known as a conjecture of Hartshorne.

Conjecture ( $c f$. Hartshorne [6]). If $X \subset \mathbb{P}^{N}$ is a non-singular closed subvariety and $\operatorname{dim} X>2 N / 3$, then $X$ is a complete intersection.

If this conjecture is true, then we obtain that every non-degenerate and non-singular variety which is degree 5 and codimension 2 is not contained in $\mathbb{P}^{N}$ for $N \geq 7$. As every non-singular variety is projectively normal if it is a complete intersection, therefore the results in this paper are recognized as a step to prove the above conjecture. Throughout this paper, variety means a complete non-singular variety. 
Notations.

$\left(D_{1} \cdots D_{n}\right)$ : The intersection number of divisors $D_{1}, \ldots, D_{n}$ on a variety $X$ where $n=\operatorname{dim} X$.

$O_{X}$ : The structure sheaf of a variety $X$.

$L_{Y}$ : The restriction of a line bundle $L$ to a subscheme $Y$.

$H^{i}(X, \mathscr{F})$ : The $i$ th cohomology group of a sheaf $F$.

$h^{i}(X, \mathscr{F})$ : The dimension of $H^{i}(X, \mathscr{F})$ as a vector space.

$|D|$ : The complete linear system defined by a divisor $D$.

$\phi_{|D|}$ : The rational map defined by $|D|$.

$\mathscr{L}$ : The invertible sheaf associated to a line bundle $L$.

$\mathscr{O}(D)$ : The invertible sheaf associated to a divisor $D$.

$\mathbb{P}(E)$ : The projective bundle defined by a vector bundle $E$.

$K_{X}$ : The canonical divisor on a non-singular variety $X$.

$\mathscr{O}_{X}(k)$ : The sheaf $\mathscr{O}_{X} \otimes \mathscr{O}_{\mathbb{P}} n(k)$ for a projective variety $X$ embedded in $\mathbb{P}^{n}$.

1. Preliminary. We give several theorems from Fujita's theory.

Definition ([2]). Let $X$ be a non-singular variety and let $L$ be an ample line bundle. We define a $\Delta$-genus of $(X, L)$ by

$$
\Delta(X, L)=\left(D^{n}\right)+n-h^{0}(X, L)
$$

where $n=\operatorname{dim} X$ and $L=\mathscr{O}(D)$.

The above pair $(X, L)$ is called a polarized non-singular variety.

DEFINITION ([8]). Let $(X, L)$ be a polarized non-singular variety. We say that $L$ is normally generated if

$$
H^{0}(X, \mathscr{L})^{\otimes k} \rightarrow H^{0}\left(X, \mathscr{L}^{\otimes k}\right)
$$

is surjective for any positive integer $k$. And in this case, we call $(X, L)$ projectively normal.

DEFINITION ([2]). Let $(X, L)$ be a polarized non-singular variety and set $L=\mathscr{O}(D)$. Let $V$ be a reduced irreducible non-singular member of $|D|$ (if there exists). We call $V$ a regular member if

$$
H^{0}(X, \mathscr{L}) \rightarrow H^{0}\left(V, \mathscr{L}_{V}\right)
$$

is surjective.

Definition ([2]). Let $(X, L)$ be a polarized non-singular variety. We define $g(X, L)$ by

$$
2 g(X, L)-2=\left(\left(K_{X}+(n-1) D\right) \cdot D^{n-1}\right)
$$

where $L=\mathscr{O}(D)$ and $n=\operatorname{dim} X$. We call this $g(X, L)$ a sectional genus of $(X, L)$. 
If $L$ is very ample, then this $g(X, L)$ is the genus of the generic curve section of $X$ in the projective embedding defined by $L$.

THEOREM A ([2]). Let $(X, L)$ be a polarized non-singular variety. If $V$ is a reduced irreducible non-singular member of $|D|$ where $\mathscr{L}=$ $\mathscr{O}(D)$, then $\Delta\left(V, L_{V}\right) \leq \Delta(X, L)$. Moreover the following conditions are equivalent:

(a) $\Delta(X, L)=\Delta\left(V, L_{V}\right)$,

(b) $V$ is a regular member.

Proof. As $0 \rightarrow \mathscr{O}_{X} \rightarrow \mathscr{L} \rightarrow \mathscr{L}_{V} \rightarrow 0$ is exact, therefore

$$
h^{0}\left(V, \mathscr{L}_{V}\right) \geq h^{0}(X, \mathscr{L})-1 \text {. }
$$

Hence $\Delta(X, L)-\Delta\left(V, L_{V}\right)=h^{0}\left(V, \mathscr{L}_{V}\right)-h^{0}(X, \mathscr{L})+1 \geq 0$, because $\left(D^{n}\right)=\left(\left.D\right|_{V^{n-1}}\right)$ where $\mathscr{L}=\mathscr{O}(D)$. By the above equation, the last part of this theorem is clear.

THeOREM B. If $X$ is a variety and $L$ is a very ample line bundle, then $\Delta(X, L) \geq 0$.

Proof. It is a well-known fact (see Fujita [1]).

Theorem C. Let $(X, L)$ be a polarized non-singular variety. If $\Delta(X, L)=0$, then $(X, L)$ is isomorphic to $\left(\mathbb{P}(E), H_{E}\right)$ or $\left(\mathbb{P}^{2}, H_{\mathbb{P}^{2}}(2)\right)$ where $E$ is a vector bundle on $\mathbb{P}^{1}, H_{E}$ is a tautological bundle on $\mathbb{P}(E)$ and $H_{\mathbb{P}^{2}}(i)=\mathscr{O}(i)$ on $\mathbb{P}^{2}(i \in \mathbb{Z})$.

Proof. This is a well-known classical theorem (see Fujita [1]).

THEOREM D ([2]). Let $(X, L)$ be a polarized non-singular variety. If $g(X, L)=0$ and $L$ is very ample, then $\Delta(X, L)=0$.

Proof. We prove this theorem by the induction on $n=\operatorname{dim} X$. If $n=1$, then this theorem is trivial. We may assume that $n \geq 2$. Let $V$ be a reduced irreducible non-singular member of $|D|$ where $\mathscr{L}=\mathscr{O}(D)$. By the induction hypothesis, we assume $\Delta\left(V, L_{V}\right)=0$ because $g\left(V, L_{V}\right)=g(X, L)=0$. Hence $H^{1}\left(V, \mathscr{L}_{V}^{\otimes(-t)}\right)=0$ for every $t \geq 0$ by Theorem $C$. Therefore the long exact sequence

$$
\cdots \rightarrow H^{1}\left(X, \mathscr{L}^{\otimes(-(t+1))}\right) \rightarrow H^{1}\left(X, \mathscr{L}^{\otimes(-t)}\right) \rightarrow H^{1}\left(V, \mathscr{L}_{V}^{\otimes(-t)}\right)
$$

says that $h^{1}\left(X, \mathscr{L}^{\otimes(-(t+1))}\right) \geq h^{1}\left(X, \mathscr{L}^{\otimes(-t)}\right)$ for any $t \geq 0$. As

$$
H^{1}\left(X, \mathscr{L}^{\otimes(-s)}\right)=0
$$


for sufficiently large $s$, we obtain $H^{1}\left(X, \mathscr{O}_{X}\right)=0$. Therefore $V$ is a regular member. Hence we obtain this theorem.

THEOREM E. Let $(X, L)$ be a polarized non-singular variety and let $d=\left(D^{n}\right)$ where $\mathscr{L}=\mathscr{O}(D)$ and $n=\operatorname{dim} X$. Moreover we assume that $\Delta(X, L) \leq g(X, L)$ and $L$ is very ample. In this case, the following are true:

(a) if $d \geq 2 \Delta(X, L)-2$, then every reduced irreducible non-singular member $V \in|D|$ is a regular member;

(b) if $d \geq 2 \Delta(X, L)+1$, then $(X, L)$ is projectively normal and $\Delta(X, L)=g(X, L)$

(c) if $d \geq 2 \Delta(X, L)+2$, then the ideal of $(X, L)$ is generated by degree 2 .

Proof. See Fujita [2]. As $L$ is very ample, the proof is the same in the case of characteristic $p>0$.

THEOREM F. Let $X \subset \mathbb{P}^{N}$ be a closed non-singular subvariety which is not contained in any hyperplane. If the degree of $X$ is 4 , then $X$ is of the following type:

(a) hypersurface,

(b) $(2,2)$ complete intersection,

(c) Segre variety $\mathbb{P}^{1} \times \mathbb{P}^{3}$ in $\mathbb{P}^{7}$,

(d) Veronese surface $\mathbb{P}^{2}$ in $\mathbb{P}^{5}$,

(e) the variety obtained by hyperplane section or projection of (a), (b), (c), (d), (e).

\section{Proof. See Swinnerton-Dyer [10].}

By the above theorems, we obtain that $(X, L)$ is projectively normal for $\left(D^{n}\right)=3,4$ where $\mathscr{L}=\mathscr{O}(D)$ and $n=\operatorname{dim} X$. Moreover $(X, L)$ is also projectively normal if $\left(D^{n}\right)=5$ and the codimension of $\phi_{|D|}(X)$ is $1,3,4$. So we consider the case that $\left(D^{n}\right)=5$ and the codimension of $\phi_{|D|}(X)$ is 2 .

2. Codimension 2 case. Throughout $\S 2$, we assume that $h^{0}(X, \mathscr{L})$ $=n+3$ where $n=\operatorname{dim} X, \mathscr{L}=\mathscr{O}(D),\left(D^{n}\right)=5$ and $L$ is very ample. In this case, $g(X, L)=1$ or 2 because $g(X, L)=0$ implies that $\Delta(X, L)=0$ by the Theorem $\mathrm{D}$. This contradicts $\left(D^{n}\right)=5$ and $h^{0}(X, L)=n+3$. If $g(X, L) \geq 2$, then $g(X, L)=2$ by Theorem $\mathrm{E}$ in $\S 1$. 
THEOREM 1. If $g(X, L)=2$, then $(X, L)$ is projectively normal and the defining ideal of $(X, L)$ is generated by degree 2 and 3 .

To prove this theorem, we prepare two lemmas.

LeMma 1. Let $(X, L)$ be as above. Let $V$ be a reduced irreducible non-singular member of $|D|$. If the homogeneous ideal of $\left(V, L_{V}\right)$ is generated by degree 2 and 3 , then the homogeneous ideal of $(X, L)$ is generated by degree 2 and 3 .

Proof. Let $I(k)$ be the polynomials defined by

$$
I(k)=\operatorname{ker}\left[S^{k} H^{0}(X, \mathscr{L}) \rightarrow H^{0}\left(X, \mathscr{L}^{\otimes k}\right)\right]
$$

where $S^{k}$ is a $k$ th symmetric product and let $I_{V}(k)$ be the polynomials defined by

$$
I_{V}(k)=\operatorname{ker}\left[S^{k} H^{0}\left(V, \mathscr{L}_{V}\right) \rightarrow H^{0}\left(V, \mathscr{L}_{V}^{\otimes k}\right)\right] .
$$

We prove this lemma by induction on $k$. In the case of $k=2,3$, this lemma is trivial. We assume that $I(k)$ is generated by $I(2)$ and $I(3)$. By Theorem E (a) in $\S 1, V$ is a regular member. Moreover $(X, L)$ and $\left(V, L_{V}\right)$ are projectively normal by Theorem $\mathrm{E}(\mathrm{b})$ in $\S 1$. Therefore we obtain the following diagram:

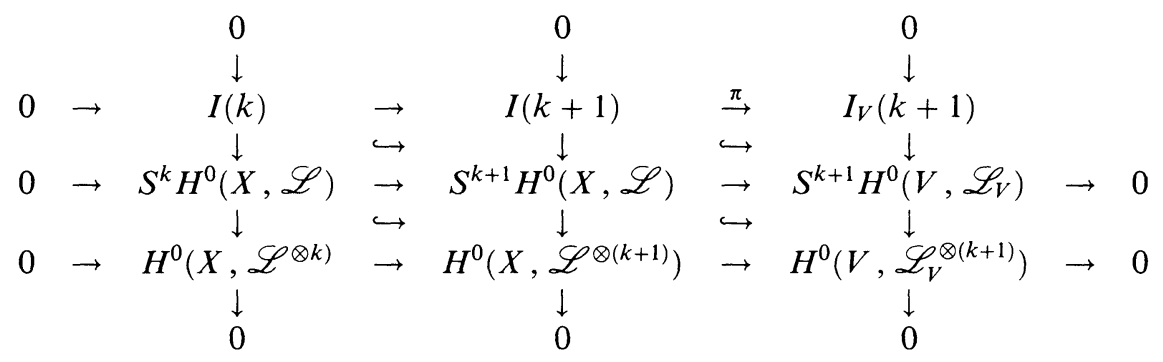

By the snake lemma, $\pi$ is a surjective map. By the assumption, $I_{V}(k+1)$ is generated by degree 2 and 3 . Therefore $I(k+1)$ is generated by degree 2 and 3 .

LEMMA 2. If $C$ is a non-singular curve and $L$ is a very ample line bundle on $C$ and $\Delta(C, L)=2$, then $(C, L)$ is projectively normal and its ideal is generated by degree 2 and 3 .

Proof. See Saint-Donat [9].

Proof of Theorem 1. It is clear by Lemma 1 and Lemma 2. 
Next we prepare the following notation.

DEFINITION. Let $(X, L)$ be a polarized non-singular variety and let $L$ be a very ample line bundle. We define $c(X, L)$ by $c(X, L)=\operatorname{minimum}\left\{i ; X=X_{n} \supset X_{n-1} \supset \cdots \supset X_{i} \supset \cdots \supset X_{1}\right.$ with

$X_{i}$ being a reduced irreducible non-singular member of $\left|D_{t+1}\right|$ where $L_{X_{T}}=\mathscr{O}\left(D_{t}\right)$ and $\left.\Delta\left(X_{n}, L_{X_{n}}\right)=\cdots=\Delta\left(X_{i+1}, L_{X_{i+1}}\right)>\Delta\left(X_{i}, L_{X_{i}}\right)\right\}$.

where $n=\operatorname{dim} X$. In the case of $\Delta\left(X_{1}, L_{X_{1}}\right)=\Delta(X, L)$, we put $c(X, L)=0$.

If $\Delta(X, L)=2$ and $g(X, L)=2$, then $c(X, L)=0$. If $\Delta(X, L)$ $=2$ and $g(X, L)=1$, then $1 \leq c(X, L) \leq \operatorname{dim} X-1$. Therefore Theorem 1 is in the case of $c(X, L)=0$.

THEOREM 2. If $c(X, L)=1$, then $(X, L)$ is projectively normal and the ideal defining $(X, L)$ is generated by degree 3.

We prepare the following two lemmas.

LEMMA 3. If $C \subset \mathbb{P}^{3}$ is a non-singular elliptic curve of degree 5 which is not contained in any hyperplane, then

$$
H^{0}\left(\mathbb{P}^{3}, \mathscr{O}_{\mathbb{P}^{3}}(k)\right) \rightarrow H^{0}\left(C, \mathscr{O}_{C}(k)\right)
$$

is surjective for every $k \geq 2$.

Proof. Let $\mathscr{O}_{C}(1)=\mathscr{O}(D)$. We obtain the following diagram:

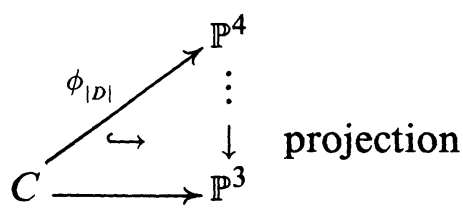

As $(C, \mathscr{O}(D))$ is projectively normal, hence

$$
H^{0}\left(C, \mathscr{O}_{C}(k)\right) \otimes H^{0}\left(C, \mathscr{O}_{C}(m)\right) \rightarrow H^{0}\left(C, \mathscr{O}_{C}(k+m)\right)
$$

is surjective for every $k, m \geq 1$. By the assumption, the canonical map

$$
H^{0}\left(\mathbb{P}^{3}, \mathscr{O}_{\mathbb{P}^{3}}(1)\right) \rightarrow H^{0}\left(C, \mathscr{O}_{C}(1)\right)
$$

is injective. Now we show that

$$
H^{0}\left(\mathbb{P}^{3}, \mathscr{O}_{\mathbb{P}^{3}}(2)\right) \rightarrow H^{0}\left(C, \mathscr{O}_{C}(2)\right)
$$


is an isomorphism. As $h^{0}\left(\mathbb{P}^{3}, \mathscr{O}_{\mathbb{P}^{3}}(2)\right)=h^{0}\left(C, \mathscr{O}_{C}(2)\right)=10$, therefore we may show that

$$
H^{0}\left(\mathbb{P}^{3}, \mathscr{O}_{\mathbb{P}^{3}}(2)\right) \rightarrow H^{0}\left(C, \mathscr{O}_{C}(2)\right)
$$

is injective. If this is not true, then there exists some quadratic surface $Q$ in $\mathbb{P}^{3}$ with $Q \supset C$. If $Q$ is non-singular, then the degree of $C=a+b$ and the genus of $C=a b-a-b+1$ for some integers $a$, $b$. This cannot occur because the degree of $C=5$ and the genus of $c=1$. If $Q$ is singular, then the genus of $C=a^{2}-a$ for odd degree $2 a+1$ of $C$. Hence degree of $C=5$ and genus of $C=1$ does not occur. Therefore the above map is injective, hence is an isomorphism. Next we show that $H^{0}\left(\mathbb{P}^{3}, \mathscr{O}_{\mathbb{P}^{3}}(3)\right) \rightarrow H^{0}(C, \mathscr{O}(3))$ is surjective. We take the basis of $H^{0}\left(C, \mathscr{O}_{C}(1)\right)$ with

$$
\begin{aligned}
H^{0}\left(\mathbb{P}^{3}, \mathscr{O}_{\mathbb{P}^{3}}(1)\right) & =\left[x_{0}, x_{1}, x_{2}, x_{3}\right], \\
H^{0}\left(C, \mathscr{O}_{C}(1)\right) & =\left[x_{0}, x_{1}, x_{2}, x_{3}, x_{4}\right]
\end{aligned}
$$

where $\left[x_{0}, \ldots, x_{N}\right]$ means that $x_{1}, \ldots, x_{N}$ are bases of a vector space. As

$$
\begin{aligned}
& H^{0}\left(\mathbb{P}^{3}, \mathscr{O}_{\mathbb{P}^{3}}(2)\right) \\
& \quad=\left[x_{0}^{2}, x_{1}^{2}, x_{2}^{2}, x_{3}^{2}, x_{0} x_{1}, x_{0} x_{2}, x_{0} x_{3}, x_{1} x_{2}, x_{1} x_{3}, x_{2} x_{3}\right]
\end{aligned}
$$

and $H^{0}\left(\mathbb{P}^{3}, \mathscr{O}_{\mathbb{P}^{3}}(2)\right) \cong H^{0}\left(C, \mathscr{O}_{C}(2)\right)$, therefore $H^{0}\left(C, \mathscr{O}_{C}(2)\right)$ has the above basis. But $x_{i} x_{4}(i=0, \ldots, 4)$ are contained in $H^{0}\left(C, \mathscr{O}_{C}(2)\right)$, and therefore we obtain the following relations:

$$
x_{i} x_{4}=f_{i}\left(x_{0}, x_{1}, x_{2}, x_{3}\right)
$$

where $i=0,1,2,3,4$ and $f_{i}(i=1,2,3,4)$ are homogeneous polynomials of degree 2 . As $\left(C, \mathscr{O}_{C}(1)\right)$ is projectively normal, hence

$$
H^{0}\left(C, \mathscr{O}_{C}(1)\right)^{\otimes 3} \rightarrow H^{0}\left(C, \mathscr{O}_{C}(3)\right)
$$

is surjective. Therefore we obtain the generators of $H^{0}\left(C, \mathscr{O}_{C}(3)\right)$ as follows,

$$
\begin{aligned}
& \left\{\begin{array}{l}
x_{0}^{3}, x_{1}^{3}, x_{2}^{3}, x_{3}^{3} \\
x_{0}^{2} x_{1}, x_{0}^{2} x_{2}, x_{0}^{2} x_{3}, x_{1}^{2} x_{0}, x_{1}^{2} x_{2}, x_{1}^{2} x_{3} \\
x_{2}^{2} x_{0}, x_{2}^{2} x_{1}, x_{2}^{2} x_{3}, x_{3}^{2} x_{0}, x_{3}^{2} x_{1}, x_{3}^{2} x_{2} \\
x_{0} x_{1} x_{2}, x_{0} x_{1} x_{3}, x_{0} x_{2} x_{3}, x_{1} x_{2} x_{3}
\end{array}\right. \\
& \left\{\begin{array}{l}
x_{4}^{3}, x_{4}^{2} x_{0}, x_{4}^{2} x_{1}, x_{4}^{2} x_{2}, x_{4}^{2} x_{3} \\
x_{4} x_{0}^{2}, x_{4} x_{1}^{2}, x_{4} x_{2}^{2}, x_{4} x_{3}^{2} \\
x_{4} x_{0} x_{1}, x_{4} x_{0} x_{2}, x_{4} x_{0} x_{3}, x_{4} x_{1} x_{2}, x_{4} x_{1} x_{3}, x_{4} x_{2} x_{3}
\end{array}\right.
\end{aligned}
$$


The part (1) is clearly the image of $H^{0}\left(\mathbb{P}^{3}, \mathscr{O}_{\mathbb{P}^{3}}(1)\right)$. And the relation $(*)$ says that the part (2) is also in the image of $H^{0}\left(\mathbb{P}^{3}, \mathscr{O}_{\mathbb{P}^{3}}(3)\right)$. Because

$$
\begin{aligned}
x_{4} x_{i} x_{j} & =f_{i}\left(x_{0}, x_{1}, x_{2}, x_{3}\right) x_{j} \quad(i, j \neq 4), \\
x_{4}^{2} x_{i} & =f_{4}\left(x_{0}, x_{1}, x_{2}, x_{3}\right) x_{i} \quad(i=0,1,2,3), \\
x_{4}^{3} & =f_{4}\left(x_{0}, x_{1}, x_{2}, x_{3}\right) x_{4}
\end{aligned}
$$

by the relation $(*)$; moreover the relation $(*)$ says $f_{4} x_{4}$ is in the image of $H^{0}\left(\mathbb{P}^{3}, \mathscr{O}_{\mathbb{P}^{3}}(3)\right)$. Hence

$$
H^{0}\left(\mathbb{P}^{3}, \mathscr{O}_{\mathbb{P}^{3}}(3)\right) \rightarrow H^{0}\left(C, \mathscr{O}_{C}(3)\right)
$$

is surjective. Finally we prove this lemma. If $k=2,3$, then this lemma is true by the above argument. We consider the case in which $k \geq 4$. First, we show this lemma in the case that $k$ is even. Let $k=2 m$. We show in this case by the induction on $m$. In this, we give the following diagram:

$$
\begin{array}{ccc}
H^{0}\left(\mathbb{P}^{3}, \mathscr{O}_{\mathbb{P}^{3}}(2 m)\right) & \rightarrow & H^{0}\left(C, \mathscr{O}_{C}(2 m)\right) \\
H^{0}\left(\mathbb{P}^{3}, \mathscr{O}(2(m-1))\right) \otimes H^{0}\left(\mathbb{P}^{3}, \mathscr{O}(2)\right) & \hookrightarrow & H^{0}(C, \mathscr{O}(2(m-1))) \otimes H^{0}(C, \mathscr{O}(2))
\end{array}
$$

By the hypothesis of induction and projective normality of $\left(C, \mathscr{O}_{C}(1)\right)$, we obtain

$$
H^{0}\left(\mathbb{P}^{3}, \mathscr{O}_{\mathbb{P}^{3}}(2 m)\right) \rightarrow H^{0}\left(C, \mathscr{O}_{C}(2 m)\right)
$$

is surjective. Next we consider the case in which $k$ is odd and $k \geq 5$. But this case is clear by the same argument. Therefore we obtain this lemma.

LEMMA 4. If $C \subset \mathbb{P}^{3}$ is as in Lemma 3, then the homogeneous ideal of $C \subset \mathbb{P}^{3}$ is generated by degree 3 .

Proof. Let $I_{k}$ be the kernel of $H^{0}\left(\mathbb{P}^{3}, \mathscr{O}_{\mathbb{P}^{3}}(k)\right) \rightarrow H^{0}\left(C, \mathscr{O}_{C}(k)\right)$. We show that

$$
I_{k} \otimes H^{0}\left(\mathbb{P}^{3}, \mathscr{O}_{\mathbb{P}^{3}}(1)\right) \rightarrow I_{k+1}
$$

is surjective for every $k \geq 3$. We take a divisor $D$ with $\mathscr{O}_{C}(1) \cong \mathscr{O}(D)$ and support of $D$ consists of 5 distinct points. As $D \subset \mathbb{P}^{2}$, we define $I_{k}^{\prime}(k=1,2, \ldots)$ by

$$
0 \rightarrow I_{k}^{\prime} \rightarrow H^{0}\left(\mathbb{P}^{2}, \mathscr{O}_{\mathbb{P}^{2}}(k)\right) \rightarrow H^{0}\left(D, \mathscr{O}_{D}(k)\right) \cong H^{0}\left(D, \mathscr{O}_{D}\right) .
$$


If $k \geq 2$, then we give the following diagram:

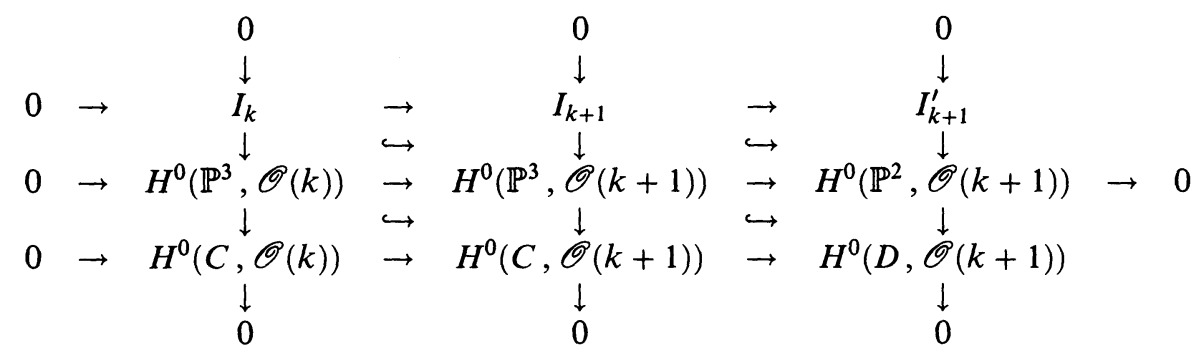

By the snake lemma,

$$
0 \rightarrow I_{k} \rightarrow I_{k+1} \rightarrow I_{k+1}^{\prime} \rightarrow 0
$$

is exact for every $k \geq 2$. Moreover we define $\lambda$ so the following diagram commutes:

$$
I_{k} \otimes H^{0}\left(\mathbb{P}^{3}, \mathscr{O}_{\mathbb{P}^{3}}(1)\right) \underset{\lambda}{\longrightarrow} I_{k}^{\prime} \otimes H^{0}\left(\mathbb{P}^{3}, \mathscr{O}_{\mathbb{P}^{3}}(1)\right)
$$

As $I_{k} \rightarrow I_{k}^{\prime}$ is surjective if $k \geq 3$ and $H^{0}\left(\mathbb{P}^{3}, \mathscr{O}_{\mathbb{P}^{3}}(1)\right) \rightarrow H^{0}\left(\mathbb{P}^{2}, \mathscr{O}_{\mathbb{P}^{2}}(1)\right)$ is surjective, therefore $\lambda$ is surjective for $k \geq 3$. Next we define $\psi: I_{k} \rightarrow I_{k} \otimes H^{0}\left(\mathbb{P}^{3}, \mathscr{O}_{\mathbb{P}^{3}}(1)\right)$ with $\psi(s)=s \otimes \delta$ where $\delta$ is a section of $H^{0}\left(\mathbb{P}^{3}, \mathscr{O}_{\mathbb{P}^{3}}(1)\right)$ which is defining $\mathbb{P}^{2}$. This shows that the following diagram

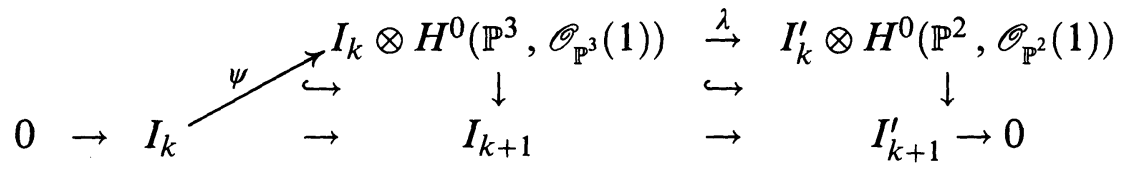

is commutative for $k \geq 2$. Therefore if $I_{k}^{\prime} \otimes H^{0}\left(\mathbb{P}^{2}, \mathscr{O}_{\mathbb{P}^{2}}(1)\right) \rightarrow I_{k+1}^{\prime}$ is surjective for every $k \geq 3$, then this lemma is proved. So we show that

$$
I_{k}^{\prime} \otimes H^{0}\left(\mathbb{P}^{2}, \mathscr{O}_{\mathbb{P}^{2}}(1)\right) \rightarrow I_{k+1}^{\prime}
$$

is surjective for $k \geq 3$. Let $V=H^{0}\left(\mathbb{P}^{2}, \mathscr{O}_{\mathbb{P}^{2}}(1)\right)$ and let $V^{k}=$ the image of $H^{0}\left(\mathbb{P}^{2}, \mathscr{O}_{\mathbb{P}^{2}}(k)\right) \rightarrow H^{0}\left(D, \mathscr{O}_{D}(K)\right)$. As the support of $D$ is not collinear, $V \rightarrow H^{0}\left(D, \mathscr{O}_{D}(1)\right)$ is injective. We show that $V^{k}=$ $H^{0}\left(D, \mathscr{O}_{D}(k)\right)$ for $k \geq 2$. If $V \neq H^{0}\left(D, \mathscr{O}_{D}(2)\right)$, then the dimension of $\operatorname{ker}\left[H^{0}\left(\mathbb{P}^{2}, \mathscr{O}_{\mathbb{P}^{2}}(2)\right) \rightarrow H^{0}\left(D, \mathscr{O}_{D}(2)\right)\right]$ is at least 2 . Therefore there exist distinct quadratics $Q_{1}$ and $Q_{2}$ with $Q_{i} \supset D(i=1,2) . Q_{1}$ and $Q_{2}$ satisfy $Q_{1} \cap Q_{2}=$ finite points. Because if $Q_{1} \cap Q_{2}$ has component, then there exist distinct lines $l_{1}, l_{2}, l_{3}$ with

$$
l_{1} \cap D=4 \text { points }
$$


and

$$
Q_{1}=l_{1}+l_{2}, \quad Q_{2}=l_{1}+l_{3} .
$$

Hence $\mathbb{P}^{3}-l_{1} \rightarrow \mathbb{P}^{1}$ be a projection with center $l_{1}$, and let $C \cdots \rightarrow \mathbb{P}^{1}$ be a restriction map to $C$. Let $f: C \rightarrow \mathbb{P}^{1}$ be an associated morphism defined by the above map $C \cdots \rightarrow \mathbb{P}^{1}$. As $l_{1} \cap D=4$ points, therefore $f$ is a bijective morphism. Hence the genus of $C=$ the genus of $\mathbb{P}^{1}=0$. This is a contradiction. So $Q_{1} \cap Q_{2}=$ finite points. As $Q_{1}$ and $Q_{2}$ are conics, $Q_{1} \cap Q_{2}$ contains at most 4 points by Bezout's theorem. But $Q_{1} \cap Q_{2}$ contains $D$ with degree 5 ; this is a contradiction. Hence $V^{2}=H^{0}\left(D, \mathscr{O}_{D}(2)\right)$. We take $s \in V$ with

$$
\begin{array}{ccc}
H^{0}\left(D, \mathscr{O}_{D}(k)\right) & \stackrel{\sim}{\rightarrow} & H^{0}\left(D, \mathscr{O}_{D}(k+1)\right) . \\
\downarrow & & \downarrow \\
t & \mapsto & t s
\end{array}
$$

In this, we obtain the following commutative diagram:

$$
\begin{array}{ccc}
H^{0}\left(\mathbb{P}^{2}, \mathscr{O}_{\mathbb{P}^{2}}(k)\right) & \rightarrow & H^{0}\left(D, \mathscr{O}_{D}(k)\right) \\
\sigma \downarrow & \hookrightarrow & \downarrow \zeta \\
H^{0}\left(\mathbb{P}^{2}, \mathscr{O}_{\mathbb{P}^{2}}(k+1)\right) & \rightarrow & H^{0}\left(D, \mathscr{O}_{D}(k+1)\right)
\end{array}
$$

where $\sigma, \zeta$, are defined by $f \mapsto f s$. Therefore we obtain

$$
H^{0}\left(\mathbb{P}^{2}, \mathscr{O}_{\mathbb{P}^{2}}(k)\right) \rightarrow H^{0}\left(D, \mathscr{O}_{D}(k)\right)
$$

is surjective if $k \geq 2$. Hence

$$
V^{k}=H^{0}\left(D, \mathscr{O}_{D}(k)\right)
$$

for every $k \geq 2$. Let $K\left(V^{k}, V\right)$ be $\operatorname{ker}\left[V^{k} \otimes V \rightarrow V^{k+1}\right]$ and $K(V, s)$ be $\operatorname{ker}\left[V^{\otimes s} \rightarrow V^{s}\right]$ where $k$ and $s$ are positive integers. We consider the following commutative diagram:

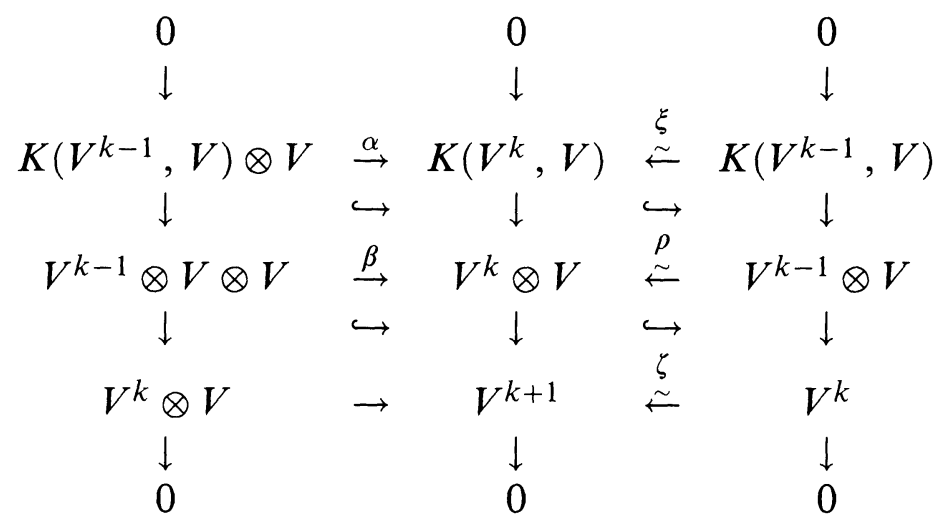


where $\beta(a \otimes b \otimes c)=a b \otimes c, \alpha$ is induced by $\beta, \zeta(f)=f s, \rho(f \otimes g)=$ $f s \otimes g, \xi$ is induced by $\rho$ and $s$ is an element of $V$ defined as above. If $k \geq 3, \rho$ and $\zeta$ are isomorphisms. Hence we obtain that $\alpha$ is a surjective map. Next we consider the following commutative diagram:

$$
\begin{array}{cccc}
0 \rightarrow K(V, k) \otimes V \stackrel{u}{\rightarrow} & K(V, k+1) & \stackrel{v}{\rightarrow} & K\left(V^{k}, V\right) \rightarrow 0 \\
w \uparrow & \hookrightarrow & \alpha \uparrow \\
& K(V, k) \otimes V & \stackrel{v^{\prime}}{\rightarrow} & K\left(V^{k-1}, V\right) \otimes V \rightarrow 0
\end{array}
$$

where $u, v, v^{\prime}$ and $w$ are canonical maps and the surjectivity of $v$ and $v^{\prime}$ is induced by the following commutative diagram and the snake lemma:

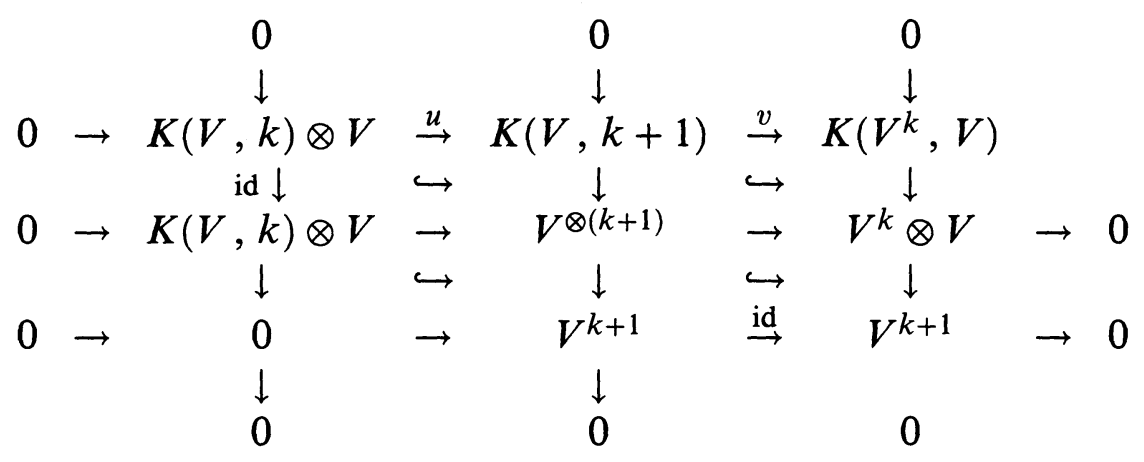

Therefore $K(V, k+1)=\operatorname{im}(w)+\operatorname{im}(u)$ if $k \geq 3$. Hence we obtain that $I_{k}^{\prime} \otimes H^{0}\left(\mathbb{P}^{2}, \mathscr{O}_{\mathbb{P}^{2}}(1)\right) \rightarrow I_{k+1}^{\prime}$ is surjective for $k \geq 3$. Hence we prove this lemma.

Proof of Theorem 2. First we show that

$$
H^{0}(X, \mathscr{L})^{\otimes k} \rightarrow H^{0}\left(X, \mathscr{L}^{\otimes k}\right)
$$

is surjective for $k \geq 1$. If $k=1$, then this is clear. Now we can take

$$
X=X_{n} \supset X_{n-1} \supset \cdots \supset X_{2} \supset X_{1}
$$

such that $X_{i}$ is a reduced irreducible non-singular member of $\left|D_{i+1}\right|$ where $\mathscr{L}_{X_{i}}=\mathscr{O}\left(D_{i}\right)(i=1,2, \ldots, n=\operatorname{dim} X)$ and

$$
2=\Delta\left(X_{n}, L_{X_{n}}\right)=\cdots=\Delta\left(X_{2}, L_{X_{2}}\right)>\Delta\left(X_{1}, L_{X_{1}}\right)=1
$$

because $c(X, L)=1$. As $X_{1}$ is an elliptic curve of degree 5 in $\mathbb{P}^{3}$, therefore $H^{0}\left(\mathbb{P}^{3}, \mathscr{O}_{\mathbb{P}^{3}}(k)\right) \rightarrow H^{0}\left(X_{1}, L_{X_{1}}^{\otimes k}\right)$ is surjective for $k \geq 2$ by Lemma 3. We consider the following diagram:

$$
\begin{aligned}
& 0 \rightarrow H^{0}\left(\mathbb{P}^{4}, \mathscr{O}(k-1)\right) \rightarrow H^{0}\left(\mathbb{P}^{4}, \mathscr{O}(k)\right) \rightarrow H^{0}\left(\mathbb{P}^{3}, \mathscr{O}(k)\right) \rightarrow 0 \\
& 0 \rightarrow H^{0}\left(X_{2}, \mathscr{L}_{X_{2}}^{\otimes(k-1)}\right) \stackrel{\hookrightarrow}{\rightarrow} H^{0}\left(X_{2}, \mathscr{L}_{X_{2}}^{\otimes k}\right) \stackrel{\hookrightarrow}{\rightarrow} H^{0}\left(X_{1}, \mathscr{L}_{X_{1}}^{\otimes k}\right)
\end{aligned}
$$


By induction on $k, L_{X_{2}}$ is projective normal. So it is clear that $L$ is projectively normal because $\Delta\left(X_{n}, L_{X_{n}}\right)=\cdots=\Delta\left(X_{2}, L_{X_{2}}\right)$. The last part of this theorem is obtained by Lemma 4 and the same argument.

COROLLARY. If $(X, L)$ is a polarized non-singular surface, $\left(D^{2}\right)=$ 5 where $\mathscr{L}=\mathscr{O}(D)$ and $L$ is very ample, then $(X, L)$ is projectively normal.

To conclude this section, we give two examples of varieties of degree 5 and codimension 2.

EXAMPLE 1. Let $f: S \rightarrow \mathbb{P}^{2}$ be a blowing up with center $p_{1}, \ldots$, $p_{8} \in \mathbb{P}^{2}$ where $p_{1}, \ldots, p_{8}$ are in general position. We put $f^{-1}\left(p_{i}\right)=$ $E_{i} \quad(i=1, \ldots, 8)$ and $D=f^{*}(4 l)-2 E_{1}-E_{2}--E_{8}$ where $l \subset \mathbb{P}^{2}$ is a line. This $D$ is very ample, $\left(D^{2}\right)=5$ and $g(S, \mathscr{O}(D))=2$ (see Hartshorne [5]). Therefore $c(S, \mathscr{O}(D))=0$.

EXAMPLe 2. Let $f: S=\mathbb{P}(\mathscr{E}) \rightarrow C$ be a ruled surface over an elliptic curve $C$ where $\mathscr{E}$ is an indecomposable locally free sheaf of rank 2 on $C$. Let $\operatorname{deg}(\mathscr{E})=1$. Let $C_{0}$ be a section of $f$ with $\operatorname{Pic}(S)=\mathbb{Z} C_{0} \oplus f^{*} \operatorname{Pic}(C)$. Let $D$ be a divisor in $\operatorname{Pic}(S)$ with $D=$ $C_{0}+f^{*}(T)$ and $\operatorname{deg}(T)=2$. This $D$ is very ample (see Hartshorne [5]). Let $l$ be a fiber of $f$. As $D$ is numerically equivalent to $C_{0}+2 l$, therefore $\left(D^{2}\right)=5$ and $\left(D \cdot\left(D+K_{S}\right)\right)=0$. Therefore $g(S, \mathscr{O}(D))=$ 1. This is an example of $c(S, \mathscr{O}(D))=1$.

\section{REFERENCES}

[1] T. Fujita, On the structure of polarized varieties with $\Delta$-genera zero, J. Fac. Sci. Univ. Tokyo, 22 (1975), 103-115.

[2] _ Defining equations for certain types of polarized varieties, in Complex Analysis and Algebraic Geometry, Iwanami (1977), 165-173.

[3] _ On the structure of polarized manifolds with total deficiency one I, II and III, J. Math. Soc. Japan, 32 (1980), 709-725, 33 (1981), 415-434, 36 (1984), 75-89.

[4] _ On polarized manifold of $\Delta$-genera two I, J. Math. Soc. Japan, 36 (1984), 709-730.

[5] R. Hartshorne, Algebraic Geometry, Springer-Verlag (1977).

[6] - Varieties of small codimension in projective space, Bull. Amer. Math. Soc., 80 (1974), 1017-1032.

[7] M. Homma, On the projective normality and defining equations of a projective curve of genus three embedded by a complete linear system, Tsukuba J. Math., 4 (1980), 269-279.

[8] D. Mumford, Varieties defined by quadratic equations, Questioni sulle varieta algebriche, Corsi dal C.I.M.E., Editoni Cremonese, Roma (1969), 29-100. 
[9] B. Saint-Donat, Sur les équations definissant une courbe algébrique, C. R. Acad. Sci. Paris Sér. A, 274 (1972), 324-327.

[10] H. P. F. Swinnerton-Dyer, An enumeration of all varieties of degree 4, Amer. J. Math., 95 (1973), 403-418.

[11] X. X. X., Correspondence, Amer. J. Math., 79 (1957), 951-952.

Received March 3, 1987.

DePaRTMENT OF MATHEMATICS

FACULTY OF EDUCATION

YAMAGUCHI UNIVERSITY

YAMAGUCHI, JAPAN 



\section{PACIFIC JOURNAL OF MATHEMATICS EDITORS}

\author{
V. S. VARADARAJAN \\ (Managing Editor) \\ University of California \\ Los Angeles, CA 90024-1555-05 \\ Herbert Clemens \\ University of Utah \\ Salt Lake City, UT 84112 \\ THOMAS ENRIGHT \\ University of California, San Diego \\ La Jolla, CA 92093
}

R. FINN

Stanford University

Stanford, CA 94305

Hermann Flaschka

University of Arizona

Tucson, AZ 85721

VAUGHAN F. R. Jones

University of California

Berkeley, CA 94720

Steven Kerckhoff

Stanford University

Stanford, CA 94305

\author{
C. C. MOORE \\ University of California \\ Berkeley, CA 94720
}

MARTIN SCHARLEMANN

University of California

Santa Barbara, CA 93106

HAROLd STARK

University of California, San Diego

La Jolla, CA 92093

\section{ASSOCIATE EDITORS}
R. ARENS
E. F. BECKENBACH
B. H. NeUmanN
F. WoLF
(1904-1989)
K. YosHIDA
(1906-1982)

\section{SUPPORTING INSTITUTIONS}

UNIVERSITY OF ARIZONA
UNIVERSITY OF BRITISH COLUMBIA
CALIFORNIA INSTITUTE OF TECHNOLOGY
UNIVERSITY OF CALIFORNIA
MONTANA STATE UNIVERSITY
UNIVERSITY OF NEVADA, RENO
NEW MEXICO STATE UNIVERSITY
OREGON STATE UNIVERSITY

UNIVERSITY OF ARIZONA

CAIVERSITY OF BRITISH COLUN UNIVERSITY OF CALIFORNIA

MONTANA STATE UNIVERSITY OREGON STATE UNIVERSITY

\author{
UNIVERSITY OF OREGON \\ UNIVERSITY OF SOUTHERN CALIFORNIA \\ STANFORD UNIVERSITY \\ UNIVERSITY OF HAWAII \\ UNIVERSITY OF TOKYO \\ UNIVERSITY OF UTAH \\ WASHINGTON STATE UNIVERSITY \\ UNIVERSITY OF WASHINGTON
}

The Supporting Institutions listed above contribute to the cost of publication of this Journal, but they are not owners or publishers and have no responsibility for its content or policies.

Mathematical papers intended for publication in the Pacific Journal of Mathematics should be in typed form or offset-reproduced (not dittoed), double spaced with large margins. Please do not use built up fractions in the text of the manuscript. However, you may use them in the displayed equations. Underline Greek letters in red, German in green, and script in blue. The first paragraph must be capable of being used separately as a synopsis of the entire paper. In particular it should contain no bibliographic references. Please propose a heading for the odd numbered pages of less than 35 characters. Manuscripts, in triplicate, may be sent to any one of the editors. Please classify according to the 1980 Mathematics Subject Classification (1985 Revision) scheme which can be found in the December index volumes of Mathematical Reviews. Supply name and address of author to whom proofs should be sent. All other communications should be addressed to the managing editor, or Elaine Barth, University of California, Los Angeles, California 90024-1555-05.

There are page-charges associated with articles appearing in the Pacific Journal of Mathematics. These charges are expected to be paid by the author's University, Government Agency or Company. If the author or authors do not have access to such Institutional support these charges are waived. Single authors will receive 50 free reprints; joint authors will receive a total of 100 free reprints. Additional copies may be obtained at cost in multiples of 50 .

The Pacific Journal of Mathematics (ISSN 0030-8730) is published monthly. Regular subscription rate: $\$ 190.00$ a year (12 issues). Special rate: $\$ 95.00$ a year to individual members of supporting institutions.

Subscriptions, orders for numbers issued in the last three calendar years, and changes of address should be sent to Pacific Journal of Mathematics, P.O. Box 969, Carmel Valley, CA 93924, U.S.A. Old back numbers obtainable from Kraus Periodicals Co., Route 100, Millwood, NY 10546.

The Pacific Journal of Mathematics at P.O. Box 969, Carmel Valley, CA 93924 (ISSN 0030-8730) is published monthly. Second-class postage paid at Carmel Valley, California 93924, and additional mailing offices. Postmaster: send address changes to Pacific Journal of Mathematics, P.O. Box 969, Carmel Valley, CA 93924.

\section{PUBLISHED BY PACIFIC JOURNAL OF MATHEMATICS, A NON-PROFIT CORPORATION}




\section{Pacific Journal of Mathematics}

Vol. 144, No. $2 \quad$ June, 1990

George E. Andrews and David M. Jackson, An algebraically derived $q$-analogue of a character sum associated with a class of semiregular

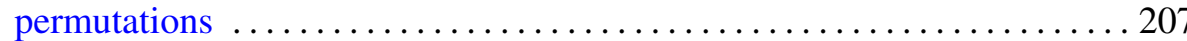

Fabio Bardelli and Andrea Del Centina, The moduli space of genus four double covers of elliptic curves is rational $\ldots \ldots \ldots \ldots \ldots \ldots \ldots \ldots 219$

Young Do Chai, An estimate of the volume of a compact set in terms of its

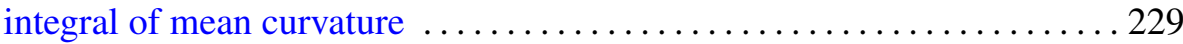

Salvador Comalada, Elliptic curves with trivial conductor over quadratic fields

Kahar El-Hussein, Résolubilité semi-globale des opérateurs différentiels invariants sur les groupes de déplacements $\ldots \ldots \ldots \ldots \ldots \ldots \ldots \ldots . \ldots 259$

David M. Goldschmidt, Classical link invariants and the Burau representation

Liliana Janicka, Radon-Nikodým problem for the variation of a vector measure

Wacław Marzantowicz, An almost classification of compact Lie groups with Borsuk-Ulam properties ............................ 299

Akira Ohbuchi, On the projective normality of some varieties of degree 5 . . 313 Ken'ichi Ohshika, Minimal measured laminations in geometric

3-manifolds

Hal Leslie Smith, A discrete Lyapunov function for a class of linear differential equations

John Samuel Spielberg, Diagonal states on $O_{2} \ldots \ldots \ldots \ldots \ldots \ldots \ldots \ldots$

Thomas Vogel, A note on the sessile drop

Gerold Wagner, On means of distances on the surface of a sphere (lower bounds) 